\title{
The diagrammatic coaction and the algebraic structure of cut Feynman integrals
}

\author{
Samuel Abreu* \\ Physikalisches Institut, Albert-Ludwigs-Universität Freiburg, D-79104 Freiburg, Germany \\ E-mail: samuel.abreulphysik.uni-freiburg.de
}

\section{Ruth Britto}

School of Mathematics, Trinity College, Dublin 2, Ireland;

Hamilton Mathematics Institute, Trinity College, Dublin 2, Ireland;

Institut de Physique Théorique, Université Paris Saclay, CEA, CNRS, F-91191 Gif-sur-Yvette

cedex, France

E-mail: ruth.britto@tcd.ie

\section{Claude Duhr}

Theoretical Physics Department, CERN, Geneva, Switzerland;

Center for Cosmology, Particle Physics and Phenomenology (CP3), Université Catholique de

Louvain, 1348 Louvain-La-Neuve, Belgium

E-mail: claude.duhr@cern.ch

\section{Einan Gardi}

Higgs Centre for Theoretical Physics, School of Physics and Astronomy, The University of Edinburgh, Edinburgh EH9 3FD, Scotland, UK

E-mail: einan.gardi@ed.ac.uk

\begin{abstract}
We present a new formula for the coaction of a large class of integrals. When applied to oneloop (cut) Feynman integrals, it can be given a diagrammatic representation purely in terms of pinches and cuts of the edges of the graph. The coaction encodes the algebraic structure of these integrals, and offers ways to extract important properties of complicated integrals from simpler functions. In particular, it gives direct access to discontinuities of Feynman integrals and facilitates a straightforward derivation of the differential equations they satisfy, which we illustrate in the case of the pentagon.
\end{abstract}

13th International Symposium on Radiative Corrections (Applications of Quantum Field Theory to Phenomenology)

25-29 September, 2017

St. Gilgen, Austria

${ }^{*}$ Speaker. 


\section{Introduction}

The realisation that a large class of Feynman integrals can be written in terms of so-called multiple polylogarithms (MPLs) has led to major advances in precision calculations in high-energy physics. A deeper understanding of the algebraic structure of MPLs has contributed to the development of new efficient techniques to evaluate Feynman integrals and to handle the complicated analytic expressions inherent to these computations, see e.g. [1,2]. In this context, a very important tool is the coaction [3, 4], a mathematical operation that exposes properties of MPLs through a decomposition into simpler functions. Starting from two loops, there are Feynman integrals that cannot be written in terms of MPLs only, and extending the coaction to more general classes of functions is a pressing question, see e.g. [5]. Here, we discuss the coaction introduced in [6] and its application to one-loop Feynman integrals where it has a simple diagrammatic representation [7]. We illustrate how it constrains the algebraic structure of these functions by discussing its implications in the study of their discontinuities and the differential equations they admit.

\section{A coaction on integrals}

In [6], a new formula for the coaction on a large class of integrals was proposed which we briefly review here. Let $\omega$ be a closed differential form and $\gamma$ a contour such that the integral of $\omega$ over $\gamma$ converges. For simplicity, we also assume that $\omega$ vanishes on the boundary of $\gamma$. We conjecture that

$$
\Delta\left(\int_{\gamma} \omega\right)=\sum_{i} \int_{\gamma} \omega_{i} \otimes \int_{\gamma_{i}} \omega .
$$

The $\omega_{i}$ are a basis of master integrands and the $\gamma_{i}$ a dual basis of master contours, in the sense that

$$
P_{s s}\left(\int_{\gamma_{i}} \omega_{j}\right)=\delta_{i j}
$$

where $P_{s s}$ is a projector onto the subspace of 'semi-simple' objects $x$ on which the coaction acts trivially, $\Delta(x)=x \otimes 1$. For the purpose of these proceeding, it is sufficient to consider $P_{s s}$ as setting to zero all polylogarithmic functions, except where they evaluate to $\pi$ or even powers of $\pi$. The second integral on the right-hand-side of eq. (2.1) is defined mod $i \pi$.

The procedure to construct a coaction based on eq. (2.1) has three main steps:

1. construct a basis of master integrands $\omega_{i}$;

2. construct a basis of master contours $\Gamma_{i}$;

3. rotate and normalise the basis $\Gamma_{i}$ to find a new basis $\gamma_{i}$ dual to $\omega_{i}$ in the sense of eq. (2.2).

In the last step we can modify the $\omega_{i}$ basis instead of the $\Gamma_{i}$, the goal being to construct dual bases.

The coaction of eq. (2.1) interacts with discontinuities and differential equations in a very simple way. Loosely speaking, the computation of a discontinuity can be seen as the modification of the integration contour $\gamma$ without modifying the integrand; conversely, differentiation modifies the integrand $\omega$ but not the integration contour. We thus conclude that discontinuities only act on the first entry of the coaction, and derivatives on the second, which can be written as:

$$
\Delta \text { Disc }=(\text { Disc } \otimes \text { id }) \Delta \quad \text { and } \quad \partial \text { Disc }=(\text { id } \otimes \partial) \Delta,
$$

where id denotes the identity operator. 


\subsection{Example: the coaction on multiple polylogarithms}

As a first example of the use of eq. (2.1), we follow the steps above to reproduce the wellknown coaction on multiple polylogarithms (MPLs) for generic values of the arguments [3, 4]. MPLs are defined iteratively as

$$
G(\vec{a} ; z)=\int_{0}^{z} \frac{d t}{t-a_{1}} G\left(a_{2}, \ldots ; t\right) \equiv \int_{0}^{z} \omega_{\vec{a}},
$$

and it is easy to see that the set of master integrands associated with the integral $G(\vec{a} ; z)$ is $\omega_{\vec{b}}$ with $\vec{b} \subseteq \vec{a}$ (we denote the empty subset as $\overrightarrow{0}$ and set $\omega_{\overrightarrow{0}}=d t$ ). The obvious candidates for master contours are the paths from 0 to $z$ that encircle a subset $\vec{b}$ of the poles in $\vec{a}$, which we denote $\Gamma_{\vec{b}}$, with $\Gamma_{\overrightarrow{0}}$ the straight line from 0 to $z$. The bases $\omega_{\vec{a}}$ and $\Gamma_{\vec{b}}$ are not dual to each other in the sense of eq. (2.2), but this can be fixed by normalising the contours as $\gamma_{\vec{b}}=c_{\vec{b}} \Gamma_{\vec{b}}$, with $c_{\vec{b}}=z^{-1}$ if $\vec{b}=\overrightarrow{0}$ and $c_{\vec{b}}=(2 \pi i)^{-|\vec{b}|}$ otherwise. Defining $G_{\vec{b}}(\vec{a} ; z) \equiv \int_{\vec{b}} \omega_{\vec{a}}$, we then obtain

$$
\Delta G(\vec{a} ; z)=\sum_{\vec{b} \subseteq \vec{a}} \int_{0}^{z} \omega_{\vec{b}} \otimes \int_{\gamma_{\vec{b}}} \omega_{\vec{a}}=1 \otimes G(\vec{a} ; z)+\sum_{\overrightarrow{0} \neq \vec{b} \subseteq \vec{a}} G(\vec{b} ; z) \otimes G_{\vec{b}}(\vec{a} ; z),
$$

which reproduces the expected coaction on MPLs [3, 4]. We have separated the term corresponding to $\vec{b}=\overrightarrow{0}$ for later reference.

\section{Coaction of one-loop (cut) Feynman integrals}

We conjecture that the coaction in eq. (2.1) applies to a large class of integrals. Here, we will show how it applies to one-loop Feynman integrals in dimensional regularisation and explore some of the consequences $[6,7,8]$. This is a very convenient choice for testing the applicability of eq. (2.1) beyond the case of MPLs. Indeed, since the coefficients of the Laurent expansion in the dimensional regulator $\varepsilon$ of any one-loop integral are conjectured to be MPLs, we can check that the prediction of eq. (2.1) for the coaction on one-loop integrals prior to expansion reproduces the combinatorics of the well established coaction on MPLs after expansion.

A basis of master integrands We consider one-loop Feynman integrals with an arbitrary configuration of internal and external masses. It is well known that integrals with different powers of the propagators satisfy integration-by-parts (IBP) relations [9], and integrals in different spacetime dimensions satisfy dimension-shift identities [10]. Tensor integrals can be reduced through Passarino-Veltman reduction [11]. Taking all these relations into account, we can choose a basis of master integrands labeled by the corresponding Feynman graph $G$ as ${ }^{1}$

$$
\omega_{G}=\frac{e^{\gamma_{E} \varepsilon} \mathscr{N}_{E_{G}}}{i \pi^{D_{G} / 2}} d^{D_{G}} k \prod_{j=0}^{\left|E_{G}\right|-1} \frac{1}{\left(k-q_{j}\right)^{2}-m_{j}^{2}}
$$

where $E_{G}$ denotes the set of internal edges of $G$ (the propagators) and we change the dimensions according to the number of propagators, $D_{G}=2\left\lceil\left|E_{G}\right| / 2\right\rceil-2 \varepsilon$. The $q_{j}$ are linear combinations of

\footnotetext{
${ }^{1}$ Strictly speaking, this is an over complete basis as for sufficiently degenerate kinematic configurations there are new IBP relations, such as the one relating the one-mass triangle to the one-mass bubble. This is not an issue for us because we work in dimensional regularisation where these limits are smooth.
} 
the external momenta $p_{i}$, and the dependence of $\omega_{G}$ on the kinematic invariants $p_{i} \cdot p_{j}$ and $m_{j}^{2}$ is implicit. $\mathscr{N}_{E_{G}}$ is a normalisation factor that guarantees that the associated Feynman integral has 'unit leading singularity' [12] and it can be computed by evaluating the maximal cut at $\varepsilon=0$. In direct analogy with the case of MPLs discussed above, the basis of master integrands relevant to the study of the Feynman integral associated with the graph $G$ is given by all $\omega_{G_{C}}$, where $C$ is a subset of $E_{G}$ and $G_{C}$ is the graph obtained by pinching all propagators that are not in $C$.

This choice of master integrands is motivated by the fact that the corresponding Feynman integrals

$$
J_{G}=\frac{e^{\gamma_{E} \varepsilon} \mathscr{N}_{E_{G}}}{i \pi^{D_{G} / 2}} \int d^{D_{G}} k \prod_{j=0}^{\left|E_{G}\right|-1} \frac{1}{\left(k-q_{j}\right)^{2}-m_{j}^{2}} \equiv \int_{\Gamma_{\emptyset}} \omega_{G}
$$

have very nice analytic properties. In particular, they satisfy differential equations in so-called canonical form [2] and the coefficients of their Laurent expansion in $\varepsilon$ have uniform weight.

A basis of master contours Any two integration contours are equivalent if one can be smoothly deformed into the other, without crossing any singularities of the integrand. To find a basis of master contours, we thus start by studying the singularities of the integrand, the so-called Landau singularities [13]. For one-loop integrals, there are two kinds of singularities: the singularities of the first kind, corresponding to a set of propagators being put on-shell, and the singularities of the second kind, which also pinch the contour at infinity. Candidates for master contours are thus the contours $\Gamma_{i_{1} \ldots i_{n}}$ that encircle the poles of propagators $i_{1}$ through $i_{n}$, and the contours $\Gamma_{\infty i_{1} \ldots i_{n}}$ which also encircle the pole at infinity. The question of whether all these contours are independent can be answered in the context of homology theory. This was addressed in the 60s [14] for integer space-time dimensions, and we have checked that the conclusions still hold in dimensional regularisation [8]. The so-called Decomposition Theorem establishes that the contours satisfy the following relations:

$$
\begin{array}{ll}
|C| \text { odd: } & \Gamma_{\infty C}=-2 \Gamma_{C}-\sum_{e \in E_{G} \backslash C} \Gamma_{C e}+\ldots, \\
|C| \text { even: } & \Gamma_{\infty C}=-\sum_{e \in E_{G} \backslash C} \Gamma_{C e}-\sum_{e, f \in E_{G} \backslash C} \Gamma_{C e f}+\ldots,
\end{array}
$$

where $C$ is a subset of the set of propagators $E_{G}$ of a graph $G$, and we have omitted writing contours that lead to contributions that vanish $\bmod i \pi$. Due to eqs. (3.3) and (3.4), we may exclude the contours that encircle the pole at infinity and choose the elements of our basis of master contours to be the $\Gamma_{i_{1} \ldots i_{n}}$, labeled by the subset of propagator poles they encircle.

This choice of master contours is directly related to the notion of cut Feynman integral. Indeed, we must evaluate integrals of the form

$$
\mathscr{C}_{C} J_{G} \equiv \int_{\Gamma_{C}} \omega_{G}
$$

which correspond to setting the propagators in $C$ on-shell. In the one-loop case, these integrals have been extensively discussed in [8]. We note that the relations in eqs. (3.3) and (3.4) directly translate to new relations between cut integrals that do not follow from IBP or dimension-shift identities. 
A dual basis of master contours Having identified a basis of master integrands and master contours, we must now make them dual to each other. For $|C|$ even, we have

$$
P_{s s}\left(\int_{\Gamma_{C}} \omega_{G}\right)=\delta_{C, E_{G}} \quad \Rightarrow \quad \gamma_{C}=\Gamma_{C}
$$

which is consistent with the fact that the normalisation factor $\mathscr{N}_{E_{G}}$ in eq. (3.1) is the maximal cut evaluated at $\varepsilon=0$. For $|C|$ odd, the relation with the dual basis is more complicated:

$$
P_{s s}\left(\int_{\Gamma_{C}} \omega_{G}+\frac{1}{2} \sum_{e \in E_{G} \backslash C} \int_{\Gamma_{C e}} \omega_{G}\right)=\delta_{C, E_{G}} \quad \Rightarrow \quad \gamma_{C}=\Gamma_{C}+\frac{1}{2} \sum_{e \in E_{G} \backslash C} \Gamma_{C e} .
$$

The reason for this more complicated relation can be understood by writing one-loop integrals as integrals over a compact quadric in the complex projective space $\mathbb{C P}^{D+1}$. Then it becomes clear that members of our basis of master integrands taken at $\varepsilon=0$ have a simple pole at infinity for $\left|E_{G}\right|$ odd, which is absent in the case of $\left|E_{G}\right|$ even. If we had allowed ourselves to pick master contours that encircle infinity for $|C|$ odd, as would be natural since this extra pole is present, then the relation with the dual basis would have been simpler. Indeed, using eq. (3.3) we have

$$
\gamma_{C}=\Gamma_{C}+\frac{1}{2} \sum_{e \in E_{G} \backslash C} \Gamma_{C e}=-\frac{1}{2} \Gamma_{\infty C}+\ldots, \quad|C| \text { odd },
$$

where the dots lead to contributions that vanish $\bmod i \pi$.

\subsection{Diagrammatic coaction for one-loop Feynman integrals}

Having constructed dual bases of master integrands and master contours, we can now use eq. (2.1) to write the coaction on one-loop Feynman integrals:

$$
\Delta\left(J_{G}\right)=\sum_{\emptyset \neq C \subseteq E_{G}} \int_{\Gamma_{\emptyset}} \omega_{G_{C}} \otimes \int_{\gamma_{C}} \omega_{G}
$$

Because master contours and master integrals can be labeled by subsets of propagators of the Feynman graph $G$, and given eq. (3.5), this coaction has a very simple diagrammatic formulation:

$$
\Delta\left(J_{G}\right)=\sum_{\emptyset \neq C \subseteq E_{G}} J_{G_{C}} \otimes\left(\mathscr{C}_{C} J_{G}+a_{C} \sum_{e \in E_{G} \backslash C} \mathscr{C}_{C e} J_{G}\right),
$$

where $a_{C}=1 / 2$ for $|C|$ odd and 0 otherwise. We note that all our discussion in this section extends trivially to the coaction on cut one-loop Feynman integrals, but we will not discuss this further in these proceedings.

\section{Checks and applications of the diagrammatic coaction}

The diagrammatic coaction of eq. (3.9), or equivalently eq. (3.10), is based on the conjecture in eq. (2.1), and we must show that it gives consistent results. We now briefly review the several checks discussed in [7]. 
The first nontrivial consistency check of eq. (3.9) is to verify that coaction components of the form $1 \otimes J_{G}$ are correctly reproduced. Indeed, the empty set is excluded from the sum in eq. (3.9) because $\Gamma_{\emptyset}$ is not a master contour, and we thus only have cut integrals appearing in the second entry of the tensor in the coaction. This is unlike what happened for MPLs where this term appears explicitly, see eq. (2.5). The only way for the coaction to be consistent is that there is a linear combination of cut integrals that reproduces the uncut integral. This relation can be obtained by setting $C=\emptyset$ in eq. (3.4), and then computing the integral over $\Gamma_{\infty}$. We then find the remarkable relation valid for any one-loop integral [8]:

$$
\sum_{e \in E_{G}} \mathscr{C}_{e} J_{G}+\sum_{\substack{e, f \in E_{G} \\ e<f}} \mathscr{C}_{e f} J_{G}=-\varepsilon J_{G} \bmod i \pi .
$$

This relation guarantees that terms of the form $1 \otimes J_{G}$ are correctly reproduced by eq. (3.9). The coaction component $J_{G} \otimes 1$ can also be checked to be correctly reproduced by eq. (3.9) for any one-loop integral.

Aside from consistency checks, we have also verified eq. (3.9) in a large number of examples. For these, it is important to stress that the coaction is valid in dimensional regularisation and prior to expansion in $\varepsilon$. This means that although we formulated it for a completely generic mass configuration, it is still valid in any degenerate kinematic configuration: one simply sets to zero scaleless Feynman integrals and vanishing cut integrals. As an example, consider a three-point function with massless propagators. The pinches include scaleless tadpoles which are set to zero, and we thus obtain from eq. (3.10):

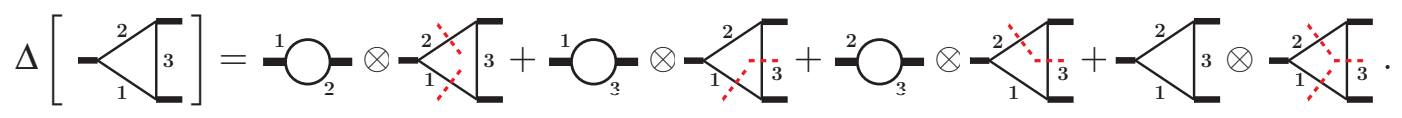

To verify that (4.2) is correct, each integral is expanded in $\varepsilon$. Each term in the expansion is a combination of MPLs, of increasing weight as one goes to higher orders in $\varepsilon$. At each order in $\varepsilon$, we check that the coaction of MPLs acting on both sides of eq. (4.2) gives the same result. Through this approach, we reduce the check of our conjecture to a check that relies on the coaction of MPLs, which was first established from completely different arguments on a rigorous mathematics footing $[3,4]$. For this example, the explicit check was done up to weight 4 . Note that while the left-hand side of eq. (4.2) is finite, the right-hand side has poles associated with the bubble integrals. The contribution of these poles cancels because of eq. (4.1). The cancellation of the poles appearing from bubble or tadpole integrals due to eq. (4.1) is another general feature of the diagrammatic coaction.

\subsection{Applications of the diagrammatic coaction}

We now briefly discuss how the diagrammatic coaction encodes the algebraic structure of oneloop integrals, and how it can be used to obtain important information on these functions in a simple way. In particular, we would like to highlight the fact that it constrains the algebraic structure of a complicated integral through relations to simpler ones, obtained from its pinches and cuts.

\subsubsection{Discontinuities}

All known results on discontinuities of Feynman integrals (see [15] for some examples) are trivially reproduced by the diagrammatic coaction, because the first entries of the coaction tensors 
are Feynman integrals, and discontinuity operators only act on the first entry of the coaction, see eq. (2.3). As an example, consider the discontinuity on the channel $s_{12}=\left(q_{1}-q_{2}\right)^{2}$ of the three point function in eq. (4.2), with $q_{i}$ the momentum of propagator $i$ and all $q_{i}$ moving clockwise around the diagram. To compute this discontinuity, one simply needs to know how to compute the discontinuity of the bubble integral appearing in the first term on the right-hand side of eq. (4.2), which is trivial as it is a single scale integral whose discontinuity is just $2 \pi i$. We directly recover the expected result that the discontinuity function is the two-propagator cut (we write $\sim$ because the precise relation depends on the definition of Disc):

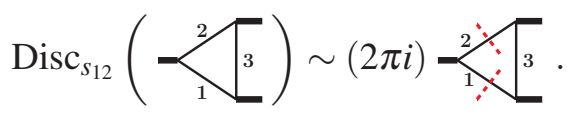

The relation between coaction entries and discontinuities, and in particular the reason why it is sufficient to consider the term with a bubble integral in the first entry for this example, is explained in more detail in [7]. The diagrammatic coaction also allows us to present a sharper version of the so-called 'first-entry-condition': In the coaction of a (cut) Feynman integral, the first entries are themselves Feynman integrals, with a subset of propagators but the same set of cut propagators.

\subsubsection{Differential equations}

Another new insight given by the diagrammatic coaction on the algebraic structure of one-loop cut Feynman integrals relates to the differential equations they satisfy. The fact that differential operators only act on the last entry of the coaction, see eq. (2.3), implies that the differential equation of any one-loop integral is completely determined from its cuts. In fact, it is determined by a very small subset of all cuts, and only at very specific orders in $\varepsilon$ [7]. More precisely, the coefficients of the differential equation are just the derivatives of the weight-one terms in the Laurent expansion of any of the cuts of the integral under consideration. For a finite one-loop integral, these are: the maximal cut at order $\varepsilon^{1}$, the next-to-maximal cut at order $\varepsilon^{1}\left(\varepsilon^{0}\right)$ for an even (odd) number of propagators, and the next-to-next-to-maximal cut at order $\varepsilon^{0}$. These cuts have been computed for a completely generic one-loop integral, and are easily written in terms of Cayley and Gram determinants (see eqs. (9.11) and (9.12) in [7]). Using the diagrammatic coaction, together with the explicit results of these cuts, we can thus write down the differential equation for any one-loop integral, without needing IBP relations as required in more standard approaches. For instance, the differential equation for a five-point function in our basis, i.e. in $6-2 \varepsilon$ dimensions and normalised to have unit leading singularity, and with any mass configuration is given by

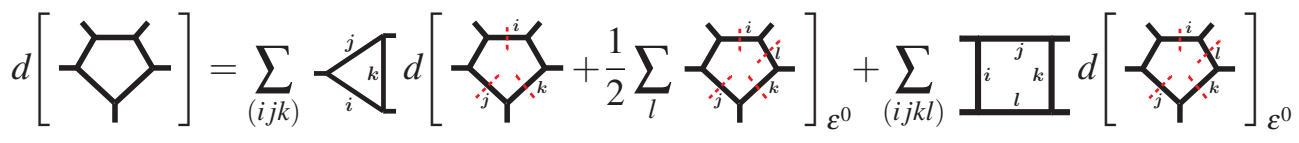

$$
\begin{aligned}
& +\varepsilon \prec-\mathcal{Y}_{\varepsilon^{1}}
\end{aligned}
$$

where $i, j, k$ and $l$ run over distinct edges of the graph. Since the knowledge of the differential equation completely determines the symbol of a function, these cuts iteratively construct the symbol of any one-loop integral (an alternative algorithm was recently proposed in [16]). This is another example of how the coaction allows us to gain information on the algebraic structure of complicated functions from that of simpler ones. 
To make the discussion more concrete, we give the explicit results for the relevant cuts of the fully massless pentagon. We will use thin lines to denote massless propagators and external legs, take all external momenta as incoming and the propagator momenta $q_{i}$, with $i \in\{1, \ldots, 5\}$, moving clockwise around the diagram. The integral is then a function of the five invariants $s_{i}=\left(q_{i}-q_{i+2}\right)^{2}$, with the indices of the $q_{i}$ understood cyclically. The order $\varepsilon^{1}$ term of the maximal cut is

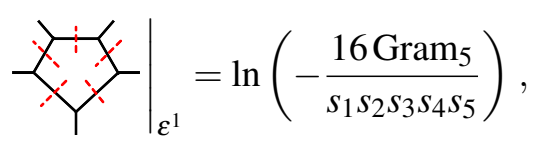

where $\operatorname{Gram}_{5}=\operatorname{det}\left(q_{i} \cdot q_{j}\right)$ for $1 \leq i, j \leq 4$ is the usual Gram determinant associated with this function. For the next-to-maximal cuts, we first define the auxiliary functions

$$
\begin{aligned}
& v\left(x_{1}, x_{2}, x_{3}, x_{4}, x_{5}\right)=x_{1} x_{5}+x_{1} x_{2}+x_{3} x_{4}-x_{2} x_{3}-x_{4} x_{5}+\sqrt{16 \mathrm{Gram}_{5}} \\
& \bar{v}\left(x_{1}, x_{2}, x_{3}, x_{4}, x_{5}\right)=x_{1} x_{5}+x_{1} x_{2}+x_{3} x_{4}-x_{2} x_{3}-x_{4} x_{5}-\sqrt{16 \mathrm{Gram}_{5}}
\end{aligned}
$$

Then, the order $\varepsilon^{0}$ term of the cut integral where all propagators but $i$ are cut is given by:

$$
\underbrace{}_{\varepsilon^{0}}=\ln \left(\frac{\bar{v}\left(s_{i-1}, s_{i}, s_{i+1}, s_{i+2}, s_{i+3}\right)}{v\left(s_{i-1}, s_{i}, s_{i+1}, s_{i+2}, s_{i+3}\right)}\right) \text {. }
$$

Finally, in the massless case all triple cuts vanish,

$$
\overbrace{Y^{*}}^{i k}=0 \quad \forall i, j, k \in\{1,2,3,4,5\} \text {. }
$$

Using eqs. (4.5), (4.7) and (4.8) in the generic expression (4.4), we obtain the differential equation for the massless pentagon, valid to all orders in $\varepsilon$. Noting that in the massless case all triangles in (4.4) are reducible to bubbles, we reproduce the expected differential equation [17]. As another check, we note that the letters in eqs. (4.5) and (4.7) are a subset of those appearing in two-loop five-point massless integrals [18].

\section{Conclusion}

We have presented a new formula for a coaction on a large class of integrals. When applied to one-loop Feynman integrals, it has a very simple diagrammatic interpretation in terms of cuts and pinches of the original integrals. The consistency of the diagrammatic coaction is checked by showing that it reproduces the combinatorics of the coaction on MPLs acting on the coefficients of the Laurent expansion of one-loop integrals. We then discussed how the diagrammatic coaction encodes important information on the algebraic structure of one-loop integrals. In particular, we argued that it reproduces known results on the discontinuities of these functions and showed how it completely determines the differential equation they satisfy. The application of the general formula for the coaction to other classes of functions, including for Feynman integrals beyond one-loop that do not evaluate to MPLs, is ongoing work. 


\section{Acknowledgments}

This work is supported by the Alexander von Humboldt Foundation in the framework of the Sofja Kovalevskaja Award 2014, endowed by the German Federal Ministry of Education and Research (SA), the ERC Consolidator Grant 647356 "CutLoops" (RB), the ERC Starting Grant 637019 "MathAm" (CD), and the STFC Consolidated Grant "Particle Physics at the Higgs Centre" (EG).

\section{References}

[1] A. B. Goncharov, M. Spradlin, C. Vergu and A. Volovich, Phys. Rev. Lett. 105 (2010) 151605, arXiv:1006.5703 [hep-th].

[2] J. M. Henn, Phys. Rev. Lett. 110 (2013) 251601, arXiv:1304.1806 [hep-th].

[3] A. B. Goncharov, arXiv:math/0208144.

[4] F. Brown, arXiv:1512.06410 [math.NT].

[5] R. Bonciani, V. Del Duca, H. Frellesvig, J. M. Henn, F. Moriello and V. A. Smirnov, JHEP 1612 (2016) 096, arXiv:1609.06685 [hep-ph]; L. Adams and S. Weinzierl, arXiv:1704.08895 [hep-ph]; J. Ablinger, J. Blümlein, A. De Freitas, M. van Hoeij, E. Imamoglu, C. G. Raab, C.-S. Radu and C. Schneider, arXiv:1706.01299 [hep-th]; J. L. Bourjaily, A. J. McLeod, M. Spradlin, M. von Hippel and M. Wilhelm, arXiv:1712.02785 [hep-th]; J. Broedel, C. Duhr, F. Dulat and L. Tancredi, arXiv:1712.07089 [hep-th].

[6] S. Abreu, R. Britto, C. Duhr and E. Gardi, Phys. Rev. Lett. 119 (2017) no.5, 051601, arXiv:1703.05064 [hep-th].

[7] S. Abreu, R. Britto, C. Duhr and E. Gardi, JHEP 1712 (2017) 090, arXiv:1704.07931 [hep-th].

[8] S. Abreu, R. Britto, C. Duhr and E. Gardi, JHEP 1706 (2017) 114, arXiv:1702.03163 [hep-th].

[9] F. V. Tkachov, Phys. Lett. B100, 65 (1981); K. Chetyrkin and F. Tkachov, Nucl.Phys. B192, 159 (1981).

[10] Z. Bern, L. J. Dixon, and D. A. Kosower, Phys. Lett. B302, 299 (1993), [Erratum: Phys. Lett.B318,649(1993)], arXiv:hep-ph/9212308 [hep-ph]; O. V. Tarasov, Phys. Rev. D54, 6479 (1996), arXiv:hep-th/9606018 [hep-th]; R. N. Lee, Nucl. Phys. B830, 474 (2010), arXiv:0911.0252 [hep-ph].

[11] G. Passarino and M. J. G. Veltman, Nucl. Phys. B 160 (1979) 151.

[12] F. Cachazo, arXiv:0803.1988 [hep-th].

[13] L. D. Landau, Nucl. Phys. 13 (1959) 181; R.J. Eden, P.V. Landshoff, D.I. Olive and J.C. Polkinghorne, The Analytic S-Matrix, Cambridge at the University Press, (1966).

[14] D. Fotiadi, M. Froissart, J. Lascoux, F. Pham, Topology 4, 159-191, Pergamon Press, (1965);

R. C. Hwa and V. L. Teplitz, Homology and Feynman integrals, W. A. Benjamin Inc., (1966).

[15] O. Steinmann, Helv.Phys.Acta. 33, 257 (1960); O. Steinmann, Helv.Phys.Acta. 33, 347 (1960); G. 't Hooft and M. J. G. Veltman, NATO Sci. Ser. B 4 (1974) 177; K. E. Cahill and H. P. Stapp, Annals Phys. 90 (1975) 438; D. Gaiotto, J. Maldacena, A. Sever and P. Vieira, JHEP 1112 (2011) 011, arXiv:1102.0062 [hep-th]; S. Abreu, R. Britto, C. Duhr and E. Gardi, JHEP 1410 (2014) 125, arXiv:1401.3546 [hep-th]; S. Abreu, R. Britto and H. Grönqvist, JHEP 1507 (2015) 111, arXiv:1504.00206 [hep-th]; S. Bloch and D. Kreimer, arXiv:1512.01705 [hep-th]; F. Brown, 
arXiv:1512.06409 [math-ph]; S. Caron-Huot, L. J. Dixon, A. McLeod and M. von Hippel, Phys. Rev. Lett. 117 (2016) no.24, 241601, arXiv:1609.00669 [hep-th].

[16] N. Arkani-Hamed and E. Y. Yuan, arXiv:1712.09991 [hep-th].

[17] M. G. Kozlov and R. N. Lee, JHEP 1602 (2016) 021, arXiv:1512.01165 [hep-ph].

[18] T. Gehrmann, J. M. Henn and N. A. Lo Presti, Phys. Rev. Lett. 116 (2016) no.6, 062001 Erratum: [Phys. Rev. Lett. 116 (2016) no.18, 189903], arXiv:1511.05409 [hep-ph]; C. G. Papadopoulos, D. Tommasini and C. Wever, JHEP 1604 (2016) 078, arXiv:1511.09404 [hep-ph]. 